\title{
Analysis of bacterial chemotactic response using dynamic laser speckle
}

Silvia E. Murialdo

Universidad Nacional de Mar del Plata

Facultad de Ingeniería

Departamento de Química

Grupo de Ingeniería Bioquímica

Juan B. Justo 4302

7600 Mar del Plata, Argentina

\section{Gonzalo H. Sendra}

Universidad Nacional de Mar del Plata

Facultad de Ingeniería

Laboratorio Láser

7600 Mar del Plata, Argentina

and

Centro de Investigaciones Opticas

(CONICET La Plata-CIC)

Casilla de Correo No 3

1897 Gonnet, La Plata, Argentina

\section{Lucía I. Passoni}

Universidad Nacional de Mar del Plata

Facultad de Ingeniería

Laboratorio de Bioingeniería

Juan B. Justo 4302

7600 Mar del Plata, Argentina

\section{Ricardo Arizaga}

Centro de Investigaciones Opticas (CONICET La Plata-CIC)

UID Optimo, Facultad de Ingeniería, Universidad Nacional de La Plata

Casilla de Correo No 3

1897 Gonnet, La Plata, Argentina

\section{J. Froilán Gonzalez}

Universidad Nacional de Mar del Plata

Facultad de Ingeniería

Departamento de Química

Grupo de Ingeniería Bioquímica

Juan B. Justo 4302

7600 Mar del Plata, Argentina

\section{Héctor Rabal}

Marcelo Trivi

Centro de Investigaciones Opticas (CONICET La Plata-CIC)

UID Optimo, Facultad de Ingeniería, Universidad

Nacional de La Plata

Casilla de Correo No 3

1897 Gonnet, La Plata, Argentina

\begin{abstract}
Chemotaxis has a meaningful role in several fields, such as microbial physiology, medicine and biotechnology. We present a new application of dynamic laser speckle (or biospeckle) to detect different degrees of bacterial motility during chemotactic response experiments. Encouraging results showed different bacterial dynamic responses due to differences in the hardness of the support in the swarming plates. We compare this method to a conventional technique that uses white light. Both methods showed to be analogous and, in some cases, complementary. The results suggest that biospeckle processed images can be used as an alternative method to evaluate bacterial chemotactic response and can supply additional information about the bacterial motility in different areas of the swarm plate assay that might be useful for biological analysis. ๑ 2009 Society of Photo-Optical Instrumentation Engineers. [DOI: 10.1117/1.3262608]
\end{abstract}

Keywords: dynamic speckle; chemotaxis; bacteria; biospeckle.

Paper 09112RR received Mar. 27, 2009; revised manuscript received Sep. 17, 2009; accepted for publication Sep. 19, 2009; published online Nov. 19, 2009.

\section{Introduction}

Chemotaxis is the directed movement of cells in response to a concentration gradient of a chemical attractant or repellent. The transport of bacterial populations through nonmixed en-

Address all correspondence to: Gonzalo Sendra, Universidad Nacional de Mar del Plata, Facultad de Ingeniería, Laboratorio de Láser, Juan B. Justo 4302, 7600 Mar del Plata, Argentina. Tel: 54-223-4816600; Fax: 54-223-481-0046; E-mail: gsendra@fi.mdp.edu.ar vironments is important in several areas, including in situ bioremediation and microbial competition. ${ }^{1}$ Chemotaxis in microorganisms has a significant role in enhancing the biodegradation of pollutants ${ }^{2}$ and an important place in the pathogenesis of enteric bacteria and Vibrio cholerae infections. ${ }^{3}$ The role of chemotaxis in Pseudomonas aerugi-

1083-3668/2009/14(6)/064015/6/\$25.00 @ 2009 SPIE 
nosa is well known; it is involved in its biofilm formation, persistence, and pathogenesis, ${ }^{4}$ as well as in adherence to solid surfaces. ${ }^{5}$ One strain of $P$. aeruginosa isolated from soil ${ }^{6}$ was used in this work for chemotactic assays due to its ubiquity and significant role in biotechnology and medicine.

More than one assay has been developed to show qualitative bacterial chemotaxis in semisolid and liquid mediums, such as agarose plug assay, swarming assay, drop assay, and capillary assay. ${ }^{7}$ In all of them, the chemotactic response is inferred by observing the spatial bacteria accumulation at different times. The swarm plate assay is a qualitative method based on the metabolism of an attractant. A carbon source (attractant) is mixed with a low percentage of agar media and poured into a transparent dish. Bacteria are stabbed in the middle of the dish and incubated. A sharp chemotactic band of bacteria growing outward is visualized in the dish, using white light, as a result of attractant metabolization and the resultant concentration gradient. ${ }^{7}$ Given its cumulative feature, this method presents advantages for long-time macroscopic observation and easy conditions of repeatability in comparison to other. However, the visible results depend on the experimental conditions and sometimes concentric nonvisible rings might not be detected.

Other optical techniques have been used for characterization of cells and bacteria motility and chemotaxis. For example, a laser-diffraction capillary assay was developed by Schmidt et al. ${ }^{8}$ to measure bacterial random motility coefficients in semisolid media. Recently, Tan et al. ${ }^{9}$ has demonstrated that it is possible to use optical coherent tomography (OCT) to evaluate dynamic cell behavior. They investigated and characterized a variety of cell processes in thick-tissue models, such as chemotaxis migration, proliferation, deadhesion, and cell-material interactions. The images obtained by the OCT technique were compared to the images obtained by confocal microscopy. Both techniques require complex and expensive equipment.

On the other hand, several biological tissues ${ }^{10-12}$ have been studied using laser speckle techniques. Nonpolished objects illuminated by laser light exhibit a visual granular appearance called speckle. This pattern is originated by the random interference of coherent wavelets coming from the scattering centers of the surface. Because of its roughness, traveling distances may differ in several wavelengths. Similar effects occur when a ground glass or other rough but transparent surface is illuminated from behind, in which case a static speckle pattern is obtained. ${ }^{13}$ If the light scattered by the surface is altered by moving particles, such as bacteria, then the obtained pattern evolves in time and it is called dynamic speckle. ${ }^{12}$ Light wavelets are rescattered by moving microorganisms in slightly different frequencies due to Doppler effects. $^{14}$

By analyzing the speckle pattern over time using dedicated algorithms, segmented images corresponding to different activity levels of the samples can be obtained. ${ }^{10,15,16} \mathrm{~A}$ computer-based acquisition system enables the recording of dynamic speckle patterns, which are postprocessed using algorithms such as generalized differences, ${ }^{16}$ wavelet entropy, ${ }^{17}$ and spectral bands decomposition. ${ }^{18}$

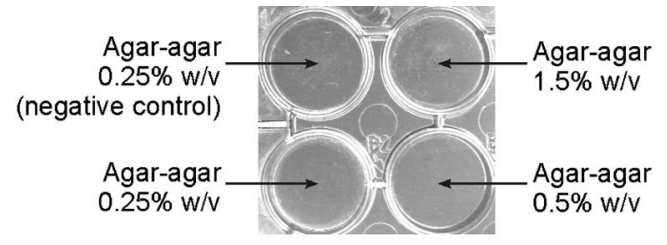

(a)

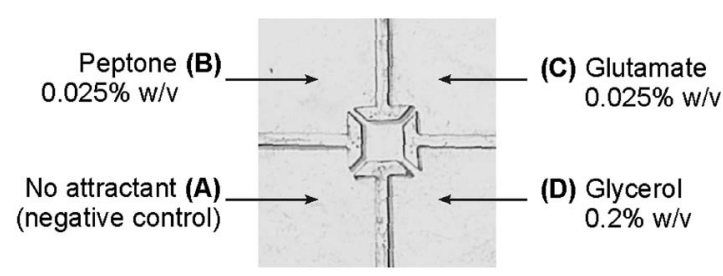

(b)

Fig. 1 (a) Wells plate photograph. Arrows indicate agar-agar concentrations. (b) Swarm plate photograph. Arrows indicate attractant concentrations.

In this work, we present the dynamic speckle activity segmentation as an alternative method to detect different degrees of motility and chemotaxis in bacteria swarming plates. The conventional white-light method detects microorganism accumulations through their optical density, without differentiating them according to their motility level. In contrast, the biospeckle technique detects microorganisms in movement. Chemotaxis response of $P$. aeruginosa toward attractants was analyzed in swarm assay with both methods.

\section{Experimental Procedures}

\subsection{Culture of $P$. aeruginosa}

A strain of $P$. aeruginosa isolated from soil ${ }^{6,19}$ was suspended into mineral salts (MS) liquid medium (composition, in grams per liter: $\mathrm{K}_{2} \mathrm{HPO}_{4}, 11.2, \mathrm{KH}_{2} \mathrm{PO}_{4}, 4.8(\mathrm{NH} 4) 2 \mathrm{SO}_{4}, 2$, $\mathrm{MgSO}_{4} \cdot 7 \mathrm{H}_{2} \mathrm{O}, \quad 0.246, \quad \mathrm{FeSO}_{4} \cdot 7 \mathrm{H}_{2} \mathrm{O}, \quad 5 \times 10^{-4}, \mathrm{pH} 7$ ) supplemented with tryptone at a final concentration of $1 \%$ $(\mathrm{w} / \mathrm{v})$. After $24 \mathrm{~h}$ of incubation at $25^{\circ} \mathrm{C}$ in an orbital shaker at $120 \mathrm{rpm}$, the cells were harvested by centrifugation at $3500 \mathrm{rpm}$ during $15 \mathrm{~min}$. Then they were washed (one time) with sterile motility buffer (MB) (10 mM potassium phosphate $\mathrm{pH} 7,0.1 \mathrm{mM}$ EDTA) and centrifuged again. The resulting precipitated cells were resuspended in MB previously vortexed to achieve good aeration. After that, the cells suspended in motility buffer, without any source of carbon and energy, were placed again in the shaker at $120 \mathrm{rpm}$ for $24 \mathrm{~h}$. Aliquots $(0.003 \mathrm{~mL})$ were used for inoculating each swarm plate in chemotaxis assays.

\subsection{Motility Assays}

We used supports with different degrees of hardness to verify if this biospeckle technique could detect bacterial motility. Four well plates were used, as described in Fig. 1(a). The wells contained MS supports with tryptone $(0.025 \% \mathrm{w} / \mathrm{v})$ as 


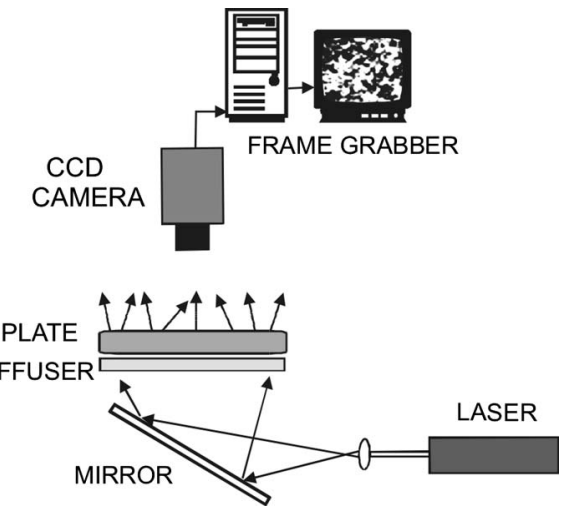

Fig. 2 Setup employed in the application of dynamic laser speckle technique to detect motility and chemotaxis in bacteria.

attractant and different concentrations of high-purity agaragar: $0.25,0.5$, and $1.5 \%(\mathrm{w} / \mathrm{v})$. The upper left well was used as a negative control in which a drop of buffer (MB) without bacteria was added. The other ones were inoculated, each one with $0.003 \mathrm{~mL}$ of cells resuspended in motility buffer.

\subsection{Swarm Plate Assays}

The swarming assay ${ }^{7,20}$ was carried out to confirm the bacterial chemotactic response. We used a swarm plate divided in four quadrants (designated as A, B, C, D) with a little central squared well [Fig. 1(b)]. The vertexes of this well were opened toward the four quadrants. Each quadrant contained soft agar-agar $(0.25 \% \mathrm{w} / \mathrm{v})$ with the following chemoattractants: (A) none (negative control), (B) peptone $(0.025 \mathrm{w} / \mathrm{v}),(\mathrm{C})$ glutamate $(0.025 \% \mathrm{w} / \mathrm{v})$, and $(D)$ glycerol $(0.2 \% \mathrm{w} / \mathrm{v})$. Harder agar-agar solution $(0.5 \% \mathrm{w} / \mathrm{v})$ was deposited inside the central well in order to slow down the interquadrant chemoeffector diffusion through the central well. The swarming assay started when aliquots of cells $(30 \mu \mathrm{L})$ resuspended in MB were inoculated into the central well. The chemoattractants in the outer quadrants spread toward the central well generating a concentration gradient necessary for the bacterial chemotactic response.

\subsection{Dynamic Laser Speckle (Biospeckle) Method}

The proposed method was implemented using the setup shown in Fig. 2. An expanded and attenuated $\mathrm{HeNe}$ laser $(632.8 \mathrm{~nm}$ and $30 \mathrm{~mW})$ illuminated the plate under study from the bottom through a ground glass diffuser. A CCD camera connected to a frame grabber registered a sequence of images ( 8 bits and $768 \times 576$ squared pixels) and stored it into the computer. A constant $4-\mathrm{Hz}$ sampling frequency was used, and the camera integration (exposure) time was set in $40 \mathrm{~ms}$.

A set of 400 sequential speckle images were acquired using the described setup. The lens of the camera was focused on the plate, and its pupil was empirically adjusted using the camera diaphragm to obtain the best spatial resolution with a speckle grain diameter comparable to the pixel size. In our case, the size of the pixel of the detector was $8.3 \times 8.3 \mu \mathrm{m}$.

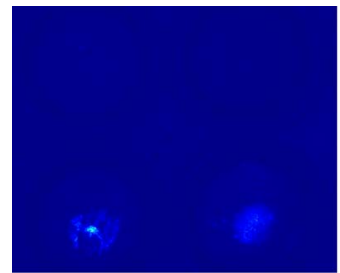

(a)

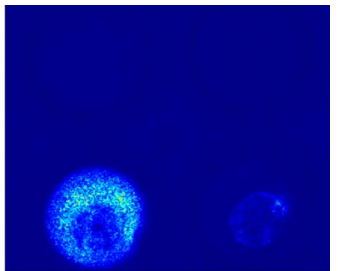

(b)
Lowest activity Highest activity

Fig. 3 Biospeckle processed images of swarm assays: (a) at $10 \mathrm{~min}$ after inoculation and (b) at $24 \mathrm{~h}$ after inoculation.

Each pixel intensity time series (signal) was considered stationary in a wide sense during the acquisition process, assuming that bacterial population movement did not exhibit relevant changes during the acquisition lapse. An infinite impulse response (IIR) bandpass digital filter was used to process these signals, and the energy of each pixel filtered time series was calculated. ${ }^{18}$ The implemented filter was a fifthorder Butterworth filter with a 1-2 Hz bandpass. To obtain an activity image, a pseudo-colored image was built where higher energy value regions stand for higher bacterial intensity movement. Finally, a spatial median filter was applied to this image to diminish granular noise, enabling better region segmentation.

\subsection{White-Light Conventional Technique}

The conventional technique to detect chemotaxis consists in observing the plate illuminated with white light from the bottom. ${ }^{20}$ Photographs were taken with the CCD camera according to the scheme of Fig. 2, where the diffuser was removed and the mirror was replaced by a circular fluorescent lamp placed over a dark surface.

\section{Results and Discussion}

As a first step to analyze dynamic speckle from bacteria, we carried out four swarming experiments with different degrees of hardness of the agar-agar [Fig. 1(a)]. It is well known that the rate of motility and chemotactic response is reduced as the hardness of the material support is increased. In Fig. 3, we present pseudocolored images obtained by the proposed method at $10 \mathrm{~min}$ [Fig. 3(a)] and $24 \mathrm{~h}$ [Fig. 3(b)] after inoculation. The bottom wells show a biological activity expansion between Figs. 3(a) and 3(b), which confirms that the activity cluster expands faster when the agar-agar hardness is reduced. On the other hand, the image zones corresponding to the well that was not inoculated with bacteria (upper left well), and the one with the hardest agar-agar (upper right well), did not present activity in comparison to the bottom wells regions [Figs. 3(a) and 3(b)].

We could conclude that speckle activity is a strong indication of local metabolic activity with displacements of bacteria. The greatest contribution to biospeckle is due to the local biological activity and the local random motion of bacteria, 


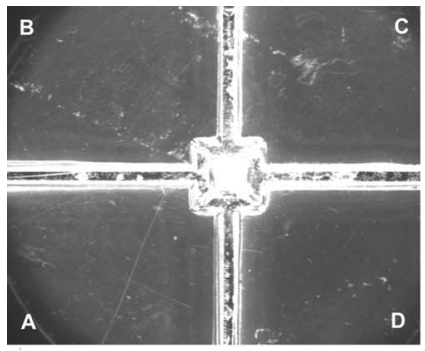

(a)

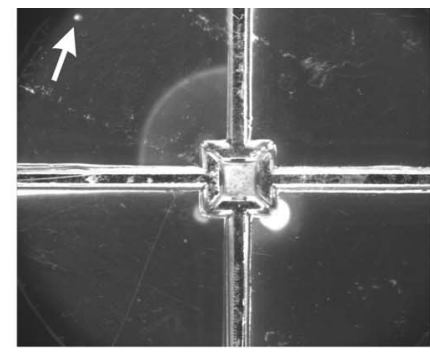

(b)

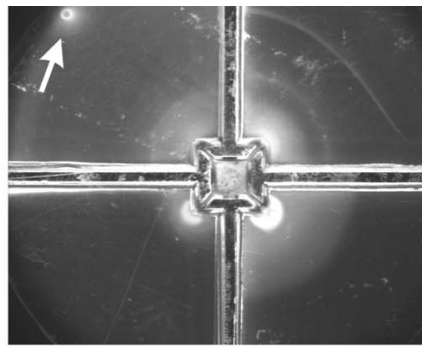

(c)

Fig. 4 White-light photograph of a swarm assay with agar-agar $(0.25 \% \mathrm{w} / \mathrm{v})$ as support and the next chemoattractans in each quadrant: (A) none (negative control), (B) peptone, (C) glutamate, and (D) glycerol. The elapsed time since inoculation with $P$. aeruginosa is (a) 10 min, (b) 21 h, and (c) $43 \mathrm{~h}$. The arrows indicate contaminations.

whereas the displacement of the cluster of bacteria, produced not only by their movement but also by their reproduction, is very slow and observed only after a few hours.

The biospeckle technique was then evaluated in a swarm plate assay. We analyzed this plate with the white-light conventional technique ${ }^{20}$ (Fig. 4) and the proposed biospeckle method (Fig. 5). The subfigures of Figs. 4 and 5 were obtained at: (a) $10 \mathrm{~min}$, (b) $21 \mathrm{~h}$, and (c) $43 \mathrm{~h}$ after inoculation. This test verified that the biospeckle and white-light images are highly correlated. For example, the presence of two rings observed with white light in quadrant $\mathrm{C}$ (glutamate) of Fig. 4(c) is validated with dynamic laser speckle image as dense zones corresponding to high activity areas, as seen in Fig. $5(\mathrm{c})$.

In Fig. 4(c), a high light-intensity region close to the vertex of the central little well was observable in the glycerol quadrant (D) using white light. However, in the processed biospeckle image [Fig. 5(c)], this small region is not clearly distinguishable. Hence, comparing both images we could infer that this small zone also contains bacteria with a very low motility level that do not contribute to the dynamic speckle response. This is an example of additional information that can provide the proposed technique when combined with the traditional one.
A small spot due to contamination was detected at $21 \mathrm{~h}$ after inoculation in the upper left quadrant of the white-light image [Fig. 4(b)]. Such a spot is almost undetectable in the corresponding biospeckle image [Fig. 5(b)]. This would mean that it belongs to a microorganism area with very low motility. Although it is also visible in Fig. 4(c) the contamination border is masked due to the presence of $P$. aeruginosa in the same area. Nevertheless, in the biospeckle image [Fig. 5(c)], after $43 \mathrm{~h}$, this contamination area was also detected and appeared to be enlarged, presenting a uniform activity level with better defined borders.

To show the differences in the information contained in the images obtained with both methods, the difference between biospeckle images and white-light images was calculated. The results, to which no quantitative meaning should be assigned because both operands represent different magnitudes, are shown in Fig. 6. In Fig. 6(a), we show the results at $21 \mathrm{~h}$ and, in Fig. 6(b), at $43 \mathrm{~h}$ after inoculation.

Dynamic speckle is produced by the beating of wavelets with Doppler-shifted frequencies. These shifts can be originated in different kinds of optical path time variations, due for example to movements, refractive index variations, configuration changes, etc. Because several of these factors are present in the behavior of bacteria with different proportions,

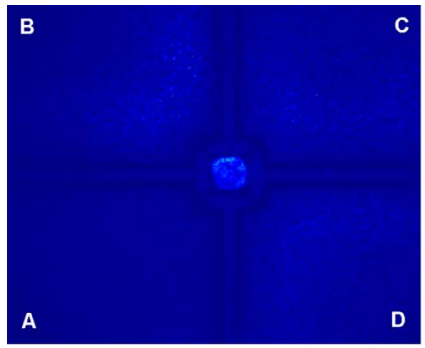

(a)

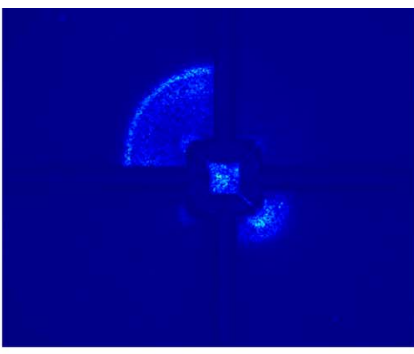

(b)

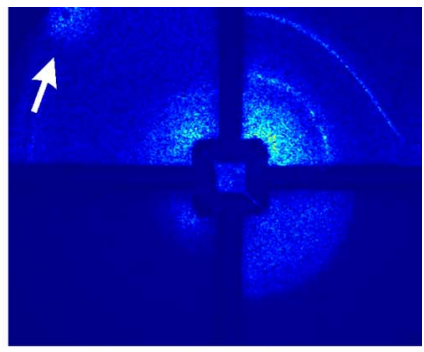

(c)

Lowest activity Highest activity

Fig. 5 Biospeckle processed images of a swarm assay with agar-agar $(0.25 \% \mathrm{w} / \mathrm{v})$ as support and the next chemoattractans in each quadrant: (A) none (negative control), (B) peptone, (C) glutamate, and (D) glycerol. The elapsed time since inoculation with $P$. aeruginosa is (a) 10 min, (b) 21 h, and (c) $43 \mathrm{~h}$. The arrows indicate contaminations. 


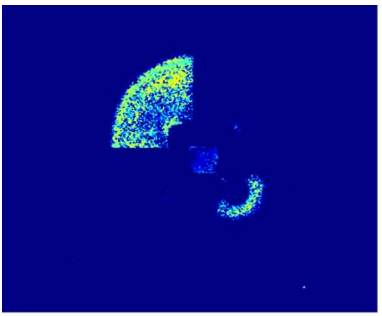

(a)

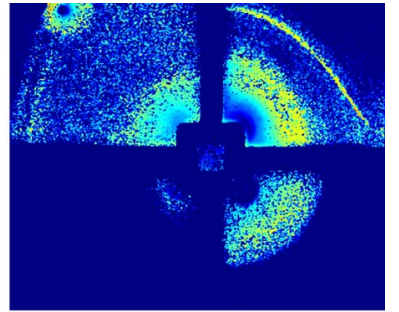

(b)
Fig. 6 Subtraction of the biospeckle image minus white light image: (a) $21 \mathrm{~h}$ after inoculation and (b) $43 \mathrm{~h}$ after inoculation. Negative results are set to zero.

the observed activity cannot be identified as due to only one of these causes.

In our case, the appearance of bright regions in the speckle images is an indication of bacterial motion as well as metabolic rate and Brownian motion. Because dead cells also exhibit Brownian motion, a contribution, albeit small, from dead cells to activity is to be expected. This contribution diminishes when the hardness of agar-agar is increased.

As an estimation, it is known that some protobacteria (as, for example, Escherichia coli) displace about $21 \mu \mathrm{m}$ per second. ${ }^{21}$ Therefore, the frequency deviation $\Delta f$ due to Doppler effect is on the order of

$$
\Delta f=\frac{1}{\lambda_{0}} n v=\frac{1}{0,633 \mu \mathrm{m}} 1.33 * 21 \frac{\mu \mathrm{m}}{\mathrm{s}}=44 \mathrm{~Hz},
$$

where $n$ is the refraction index and $v$ is the bacteria velocity. This shift frequency is equivalent to a period of $T=0.023 \mathrm{~s}$, which is about a half the integration time of the detector (40 ms), because the sampling rate is $4 \mathrm{~Hz}$. The wave fluctuates about 10 times during the sampling period. Only slow variations are detected by the camera.

The change in instantaneous frequency due to internal (metabolic) movements inside the cell should also be added to this value by introducing small time variations in the local refractive index $n$

$$
\Delta f^{\prime}=\frac{1}{\lambda_{0}} e \frac{d n}{d t}
$$

where $e$ is the typical thickness of the region where the refractive index is changing in time. The use of faster and more sensitive cameras could be useful to distinguish the different contributions.

On the other hand, incoherent illumination produces images where the contributions due to different cells are added on an intensity basis. The signal is then proportional to the local cell density. The cell's state of motion is not taken into account. Note that [Fig. 4(a)-4(c)] small (static) scratches in the support produce scattered bright signals that are formally undistinguishable from cells and that are not visible in the speckle processed images.

It can be seen that both signals represent different phenomena and could be interpreted as complementary in a certain sense. In both methods, the white-light standard technique and the dynamic laser speckle provide an image where the intensity of each pixel is proportional to the local density of bacteria. However, the main difference between them is in the ability of the second one to detect only motile bacteria clusters, while the first one does not differentiate between motile and nonmotile bacteria.

Hence, we can say that the processed full-field biospeckle images are complementary to the conventional white-light images because together both may help to the discrimination between motile and nonmotile bacteria zones. Note the difference, for example, in the lower right corner in Figs. 4 and 5. The incoherently illuminated example shows saturated regions that are not present in the speckle image. Therefore, we expect that this technique will not only be an alternative method to detect chemotaxis but also a useful tool for future microbiological research.

The processing of laser speckle frames requires simple and fast algorithms, and has a lower computational requirement as compared to OCT. $^{9}$ Also, it could, by means of special equipments, be applied in situ.

In future works, we will try to extend the use of this technique to other chemotactic assays, such as agarose plug assay and drop assay. ${ }^{7}$ These assays require a fast acquisition system because the complete process takes only a few minutes.

\section{Acknowledgments}

This work was supported by grants from Consejo Nacional de Investigaciones Científicas y Técnicas (CONICET), Comisión de Investigaciones Científicas de la Provincia de Buenos Aires (CICBA), Secretaría de Estado de Ciencia y Técnica (SECYT), Universidad Nacional de Mar del Plata (UNMDP), and Universidad Nacional de La Plata (UNLP).

\section{References}

1. R. M. Ford, "Mathematical modeling and quantitative characterization of bacterial motility and chemotaxis," in Modeling the Metabolic and Physiologic Activities of Microorganisms, C. J. Hurst, Ed., Wiley, Hoboken, NJ, pp. 177-215 (1992).

2. S. K. Samanta, B. Bhushan, A. Chauhan, and R. K. Jain, "Chemotaxis of a Ralstonia sp. SJ98 toward different nitroaromatic compounds and their degradation," Biochem. Biophys. Res. Commun. 269, 117-123 (2000).

3. S. Mariconda, Q. Wang, and R. M. Harshey, "A mechanical role for the chemotaxis system in swarming motility," Mol. Microbiol. 60 , 1590-1602 (2006).

4. G. L. Mark, J. M. Dow, P. D. Kiely, H. Higgins, J. Haynes, C. Baysse, A. Abbas, T. Foley, A. Franks, J. Morrissey, and F. O'Gara, "Transcriptome profiling of bacterial responses to root exudates identifies genes involved in microbe-plant interactions," Proc. Natl. Acad. Sci. U.S.A. 102, 17454-17459 (2005).

5. G. A. O'Toole and R. Kolter, "Flagellar and twitching motility are necessary for Pseudomonas aeruginosa biofilm development," Mol. Microbiol. 30, 295-304 (1998).

6. S. E. Murialdo, R. Fenoglio, P. M. Haure, and J. F. González, "Degradation of phenol and chlorophenols by mixed and pure cultures," Water SA 29, 457-463 (2003).

7. R. Singh and M. S. Olson, "Application of bacterial swimming and chemotaxis for enhanced bioremediation," in Emerging Environmen tal Technologies, V. Shah, Ed., Springer, New York, pp. 149-172 (2008).

8. S. Schmidt, M. T. Widman, and R. M. Worden, "A laser-diffraction capillary assay to measure random motility in bacteria," Biotechnol. Tech. 11, 423-426 (1997). 
9. W. Tan, A. L. Oldenburg, J. J. Norman, T. A. Desai, and S. A. Boppart, "Optical coherence tomography of cell dynamics in threedimensional tissue models," Opt. Express 14, 7159-7171 (2006).

10. J. D. Briers and S. Webster, "Laser speckle contrast analysis (LASCA): a nonscanning, full-field technique for monitoring capillary blood flow," J. Biomed. Opt. 1, 174-179 (1996).

11. V. V. Tuchin, Tissue Optics: Light Scattering Methods and Instruments for Medical diagnosis. SPIE, Bellingham, WA (2000).

12. J. A. Pomarico, H. O. Di Rocco, L. Alvarez, C. Lanusse, L. Mottier, C. Saumell, R. Arizaga, H. Rabal, and M. Trivi, "Speckle interferometry applied to pharmacodynamic studies: evaluation of parasite motility," Eur. Biophys. J. 33, 694-699 (2004).

13. J. C. Dainty, Laser Speckle and Related Phenomena, Springer-Verlag, Berlin (1984).

14. H. Z. Cummins and H. L. Swinney, "Light beating spectroscopy," Prog. Opt. 8, 133-200 (1970).

15. A. K. Dunn, A. Devor, H. Bolay, M. L. Andermann, M. A. Moskowitz, A. M. Dale and D. A. Boas, "Simultaneous imaging of total cerebral hemoglobin concentration, oxygenation, and blood flow during functional activation," Opt. Lett. 28, 28-30 (2003).

16. R. Arizaga, N. L. Cap, H. Rabal, and M. Trivi, "Display of local activity using dynamical speckle patterns," Opt. Eng. 41, 287-294 (2002).

17. L. I. Passoni, A. Dai Pra, H. Rabal, M. Trivi, and R. Arizaga, "Dynamic speckle processing using wavelets based entropy," Opt. Commun. 246, 219-228 (2005).

18. G. H. Sendra, R. Arizaga, H. Rabal, and M. Trivi, "Decomposition of biospeckle images in temporary spectral bands," Opt. Lett. 30, 1641$1643(2005)$

19. E. A. Wolski, S. E. Murialdo, and J. F. González, "Effect of pH and inoculum size on pentachlorophenol degradation by Pseudomonas sp," Water SA 32, 93-98 (2006).

20. J. B. Armstrong, J. Adler, and M. M. Dahl, "Nonchemotactic mutants of Escherichia coli," J. Bacteriol. 93, 390-398 (1967).

21. H. C. Berg, "Motile behavior of bacteria," Phys. Today 53, 24-29 (Jan. 2000). 